\title{
Discernable differences in the genetic and molecular profile of cerebellar glioblastoma
}

\author{
Muhibullah S. Tora ${ }^{1}$, D. Cory Adamson ${ }^{1,2,3}$ \\ ${ }^{1}$ Department of Neurosurgery, Emory University School of Medicine, Atlanta, GA, USA; ${ }^{2}$ Section of Neurosurgery, Atlanta VA Health Care System, \\ Decatur, GA, USA; ${ }^{3}$ Neuro-oncology Lab, Department of Neurosurgery, Emory University School of Medicine, Atlanta, GA, USA \\ Correspondence to: D. Cory Adamson, MD, PhD. Department of Neurosurgery, Emory University, Atlanta, GA, USA. Email: cory.adamson@emory.edu. \\ Comment on: Cho HJ, Zhao J, Jung SW, et al. Distinct genomic profile and specific targeted drug responses in adult cerebellar glioblastoma. Neuro \\ Oncol 2019;21:47-58.
}

Submitted Jul 08, 2019. Accepted for publication Aug 19, 2019.

doi: $10.21037 /$ tcr.2019.08.24

View this article at: http://dx.doi.org/10.21037/tcr.2019.08.24

\section{Introduction}

Glioblastoma (GBM) represents the most common primary malignancy of the central nervous system in adults. While the vast majority of GBMs are supratentorial lesions (S-GBM), $0.9 \%$ to $3.4 \%$ of GBMs occur in the cerebellum (C-GBM) (1-3). Considering this rarity, relatively few studies have examined C-GBMs specifically and the majority of these studies have been understandably limited by small patient populations. Despite sharing many histopathologic characteristics, the question of what degree of clinical and biological similarity there is between C-GBM and S-GBM has been raised (4). In addition, much of the molecular understanding of GBM is restricted to the supratentorial setting. Pursuant to this, studies have highlighted the need for genetic and molecular characterization of C-GBMs (3-8). In the present commentary, we will provide a brief literature review of the clinical demographics and outcomes of C-GBM. In addition, we will highlight and discuss the findings in Cho 2019 and other studies regarding the distinct genetic and molecular profile of C-GBM (8).

\section{From past to present: a brief literature review of cerebellar GBM}

Given that only a small percentage of GBMs occur in the cerebellum, it stands to reason that these lesions may be clinically distinct. As such, many studies in the past several decades have attempted to define the outcomes of patients with C-GBM. An overview of eleven highlighted studies and summary findings are presented in Table 1. Four single-arm observational studies and one meta-analysis from 1998 to 2012 report median OS between 9.9 to 18.4 months $(7,10-13)$. The three largest observational studies from Adams 2012, Babu 2013, and Cho 2019 detected no statistically significant difference in overall survival for C-GBM vs. S-GBM $(3,5,8)$. The largest study examining clinical outcomes at present by our group reported on $201 \mathrm{C}-\mathrm{GBM}$ and 36,893 S-GBMs and found a median survival of 7 months and 8 months respectively with no statistically significant difference $(\mathrm{P}=0.24)(3)$. With the increased availability of multi-institutional databases, a re-examination of these findings may be warranted. Nonetheless, there is at present no statistically significant difference in overall survival between GBMs occurring either in the supratentorial or cerebellar regions.

Few, if any, discernable demographic characteristics have been found in relation to C-GBM as compared to S-GBM. A statistically significant younger-age of diagnosis has been reported in the two largest studies in C-GBM compared to S-GBM $(3,5)$. Similar to S-GBM, these two studies also reported that younger age was a favorable prognostic factor in C-GBM patients less than 40 years of age $(3,5)$. It should be noted that both studies utilized the SEER database, but with differences in the dates of enrollment and the overall number of patients. Adams et al. also reported a predilection for Asian or Pacific Islander race, but this association was not subsequently found by Babu et al. Treatments that may confer an OS or progression-free survival (PFS) benefit have also been reported for C-GBM. Babu et al. reported that radiation therapy (HR: 0.33; 95\% CI: $0.24-0.47$; $\mathrm{P}<0.0001$ ) 
Table 1 Overview of 11 studies on cerebellar GBM reporting clinical or genetic and molecular features

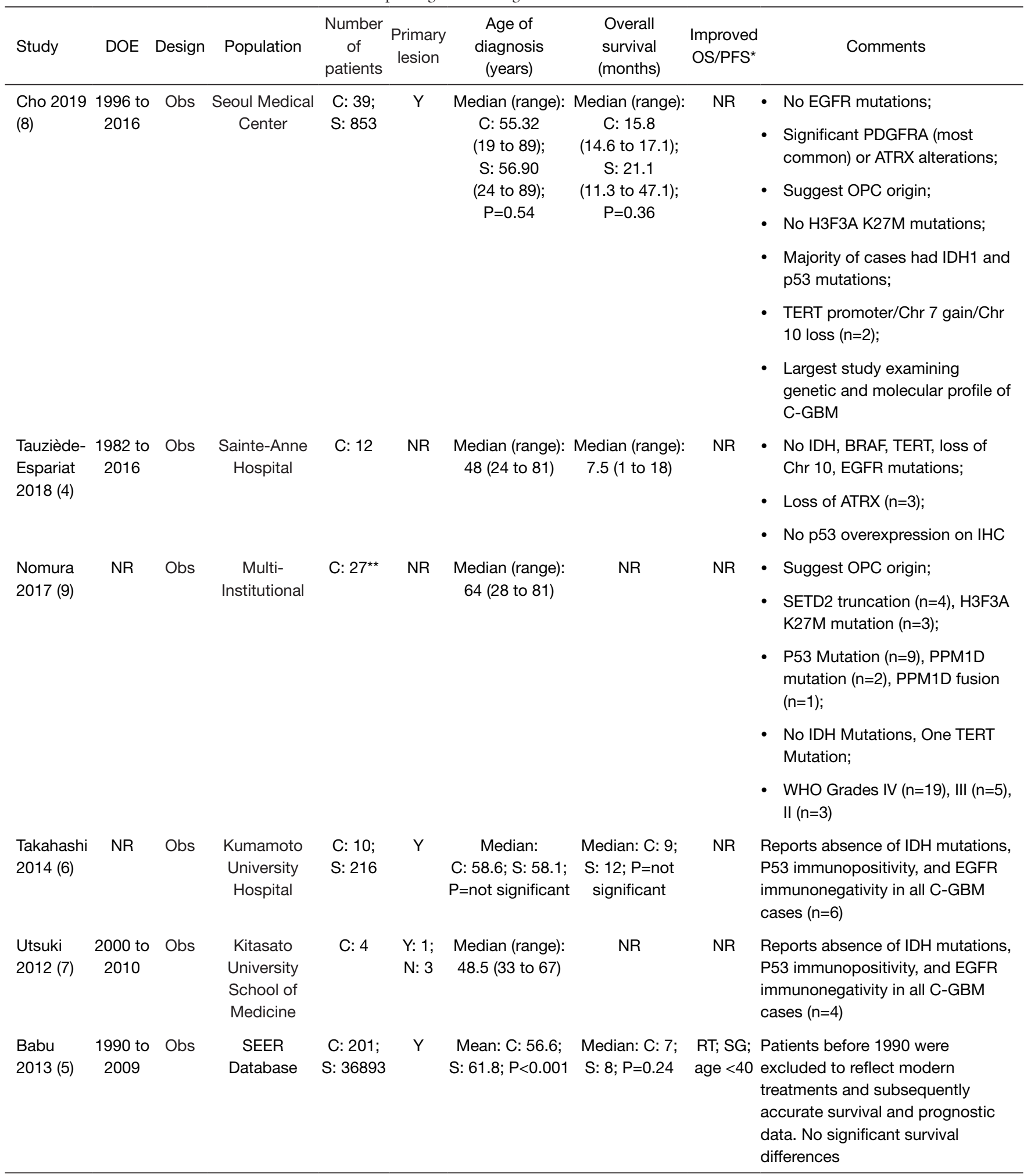

Table 1 (continued) 
Table 1 (continued)

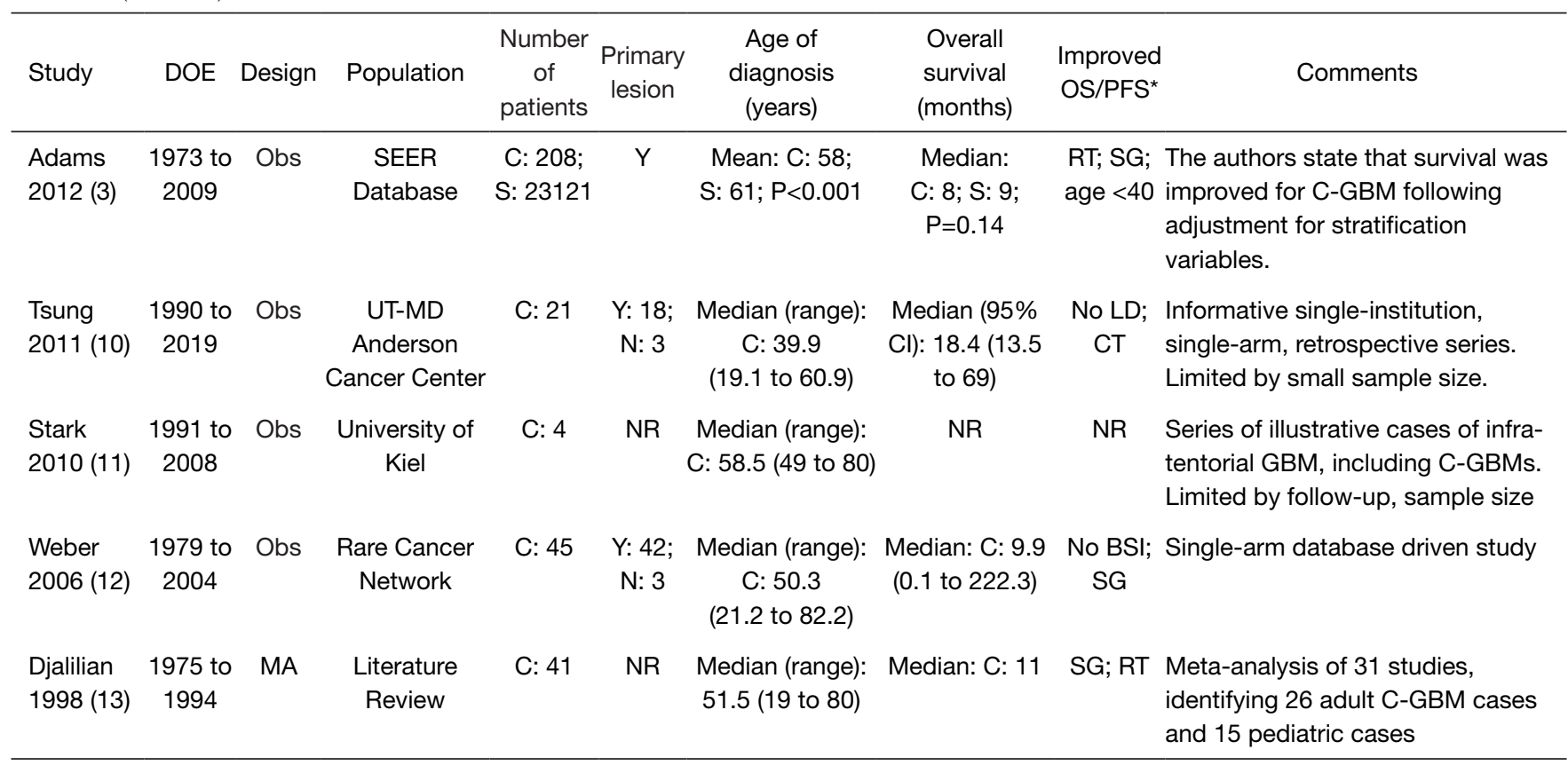

*, clinical features associated with statistically significant improved survival metrics; **, included WHO tumors less than Grade IV. DOE, dates of enrollment; $n$, number of patients; OS, overall survival; PFS, progression-free-survival; Obs, observational; MA, meta-analysis; $Y$, yes; N, no; C, cerebellar glioblastoma; S, supratentorial glioblastoma; NR, not reported; SG, surgery; RT, radiotherapy; CT, chemotherapy; $\mathrm{BSI}$, brainstem involvement; LD, leptomeningeal disease.

and surgical resection (HR: 0.66; 95\% CI: 0.45-0.96; $\mathrm{P}=0.028$ ) were independently favorable for prognosis (5). The most consistent treatment-related finding across all studies is a statistically significant survival benefit related to a greater extent of resection (3,5,10-13). Survival benefits were also reported in three studies associated with radiation therapy $(3,5,13)$, and in only one study associated with chemotherapy (10).

Overall, the clinical course of patients with C-GBM is poor and is similar to patients with S-GBM. There is at present no statistically significant difference in survival, few identifiable demographic differences, and a similar response to treatment. This suggests that both C-GBM and S-GBM are clinically similar disease entities.

\section{Distinct genetic and molecular profile of S-GBM and C-GBMs}

The clinical similarities of these lesions do not preclude potential immunohistochemical, genetic, and molecular differences in C-GBMs as compared to S-GBMs. Here we discuss five studies in the last decade which have all highlighted potential differences, outlined in Table 1 (4,6-9).
In 2012, Utsuki et al. reported four cases of C-GBM which were immuno-positive for $\mathrm{p} 53$ and immuno-negative for EGFR and IDH1, in contrast to many S-GBMs (7). Interestingly, three of the four cases also found small regions of low-grade histology within the tumors, suggesting that these C-GBMs may have arose as secondary tumors despite no detection of IDH1 and IDH2 mutations. Utsuki et al. conclude by stating that C-GBMs may arise through different mechanistic pathways as compared to S-GBMs (7). In 2014, Takahashi et al. reported similar findings in six cases of C-GBM again with p53 immunopositivity and EGFR immuno-negativity on immunohistochemistry. In addition, they noted the absence of IDH mutations with PCR amplification and sequencing. Given the limited sample size of these two studies and no further immunohistochemical or genetic characterizations, it is difficult to draw conclusions regarding the generalizability of their findings. However, they nonetheless highlight a potential difference in C-GBMs compared to S-GBM.

More recently, in 2017, Nomura et al. reported a detailed analysis of the genetic, epigenetic, and molecular profile of C-GBMs in combination with lower WHO grade cerebellar gliomas (9). They highlighted SETD2, PPM1D, 
and PDGFRA-related genetic and epigenetic changes in C-GBM compared to S-GBM, p53 mutations, infrequent EGFR mutations as well as a potential oligodendroglial progenitor cell (OPC) origin of development (9). In 2018, Tauziède-Espariat et al. examined the histo-molecular features of 12 adult C-GBMs, including IDH1/2, H3F3A/ HIST1H3B, BRAFV600E, ATRX, p16, p53, and EGFR (4). No cases had detectable IDH or BRAF mutations. In S-GBM, a common occurrence is the presence of hTERT mutations (up to $80 \%$ of cases), loss of chromosome 10 (up to $70 \%$ of cases), and EGFR amplification/gainof-function (up to $55 \%$ ) (4). Notably, none of the 12 C-GBM cases in Tauziède-Espariat et al. demonstrated any of these genetic alterations. One of 12 C-GBM cases demonstrated PDGFRA amplification. Three C-GBM cases demonstrated loss of ATRX, without a mutation in IDH/ $\mathrm{H} 3 \mathrm{~F} 3 \mathrm{~A} / \mathrm{HIST} 1 \mathrm{H} 3 \mathrm{~B}$, and without overexpression of $\mathrm{p} 53$ on immunohistochemistry. The authors conclude by stating that their results suggest that C-GBMs do not present with the same genetic lesions as the S-GBM counterparts (4).

The 2019 paper by Cho et al. is at present the largest and most detailed study examining the genetic, epigenetic, and molecular profile of C-GBMs in 19 cases of C-GBM (8). First, the authors report no significant differences in demographic features, treatment regimens, histologic features, or survival between cases of C-GBM $(n=39)$ and S-GBM ( $\mathrm{n}=814)$; which is consistent with prior studies. The authors then utilized DNA-sequencing, RNA-sequencing, and DNA methylation arrays on 19, 6, and 4 C-GBM cases respectively and made comparisons to institutional or publicly available datasets of S-GBM. Consistent with prior studies, no EGFR activating alterations were found in any cases. In addition, their data demonstrated a statistically significant increase in exclusive alterations in ATRX or PDGFRA in C-GBMs compared to S-GBMs. Four of nineteen cases had ATRX mutations, and three of these were accompanied by IDH1 and p53 wildtype in contrast to S-GBM. The majority of their C-GBM cases did in-fact have IDH1 and p53 mutations, in contrast to a few of the earlier studies. It should be noted however, that some prior studies primarily focused on dichotomous p53 immunopositivity or negativity. They also found that three of nineteen cases reported RAS mutations or amplifications, uncharacteristic of S-GBMs (8). While TERT promoter mutations with chromosome 7 gain and chromosome 10 loss are frequent in IDH wildtype S-GBMs (up to $80 \%$ of cases), the authors found only $10.5 \%$ of cases had these mutations.
In examination of GBM subtyping, they found that five of six C-GBMs which underwent subtyping were of the pro-neural subtype and had transcriptomic similarity to pro-neural S-GBM. Unfortunately, other cases were not submitted to this analysis. They also reported that despite several differences in the above mutations, transcriptomic data suggested that C-GBMs are more similar in expression pattern to S-GBMs than other infra-tentorial tumors including medulloblastoma, ependymoma, and pilocytic astrocytoma. Even so, they subsequently examined locationindependent and location-dependent gene expression levels through transcriptomic analysis. Notably, they identified that NG2 (also known as CSPG4) was upregulated in C-GBMs compared to S-GBMs. This is in agreement with the findings of Nomura et al. in 2017 who also suggested that C-GBMs may have arisen from an OPC origin (8). Cho et al. also performed DNA methylation profiling of four C-GBMs. They report specific methylation patterns in C-GBMs and in contrast to the Nomura study, Cho et al. did not find HF3A K27M mutations albeit in a limited subset of cases $(8,9)$. While this is not the only difference in findings between Cho et al. and others, there may indeed be a high degree of inter-tumor heterogeneity in C-GBM that may explain these findings (8).

Overall, Cho et al. presents several differences in this particular population of C-GBM cases compared to S-GBM (8). This is in support of the assertion that C-GBMs, while clinically similar, have genetic, epigenetic, and molecular differences that should be taken into consideration. Pursuant to this, the Cho 2019 study also conducted drug screens on C-GBM primary cells from 3 patients compared to S-GBM cells from 20 patients. Interestingly, they report that C-GBM cells were less responsive to EGFR targeted drugs, more responsive to MEK inhibitors, and more responsive for VEGFR and Ab1 inhibitors owing to their off-target inhibition of PDGFR (8). One final notable finding of Cho et al. is the specific identification of NR4A1 as a potential target in GBMs, and especially in C-GBMs as compared to S-GBM (8). Modulation of the NR4A1 family of nuclear receptors may be a potential strategy for treatment in C-GBM and other GBMs using targeted small molecules or viral gene expression, but this remains the subject of further preclinical investigation $(8,14,15)$.

\section{Future directions}

The findings by Cho et al. represent a novel step forward 
in highlighting the genetic characteristics of C-GBM as compared to S-GBM. It is our opinion that the most notable finding is the identification of NR4A1 as a potential target for therapeutic intervention. One main criticism, however, is that the vast majority of studies on C-GBM have been limited to retrospective, single-institution and observational studies that have been limited in the number of enrolled patients (Table 1). Even Cho et al. use smaller subsets of patients for their subsequent analyses. This is not surprising, given the rarity of C-GBM as compared to its supratentorial counterpart. Ideally, the next step would be a multi-institutional effort to bank tissue and generate the detailed findings as presented by Cho et al., but in a larger context. Such an approach would represent a dataset that would be more generalizable and better powered to elucidate both the genetic profile of C-GBM and respective potential targets. This could facilitate the development of personalized therapeutic approaches or, at the very least, identify more pathways of interest for pre-clinical therapeutic development. A multi-center database can also promote the improvement of non-invasive diagnostic approaches including MRI and MR-spectroscopy, as has been performed using The Cancer Genome Atlas $(16,17)$. Overall, we believe that the next major step in the field of C-GBM will require a multi-institutional and cooperative effort to produce more generalizable findings.

\section{Conclusions}

While the clinical outcomes of C-GBMs and S-GBMs are similar as reported in numerous studies, these tumors appear to have several genetic and molecular differences that must be taken into consideration in the pre-clinical setting for mechanistic study and therapeutic development. Nonetheless, there appears to be intertumoral heterogeneity in C-GBM based on the findings of Cho et al. in comparison to prior studies. Whether in the supratentorial or cerebellar setting, in accordance with many others in the field, it is our opinion that one of the most important points to take into consideration is the inter and intra-tumoral heterogeneity of GBMs in the research and clinical settings. This is well illustrated with the rise of molecular subtyping and the use of bulk and single cell transcriptomic profiling that have highlighted inter and intra-tumoral heterogeneity-where a single tumor may have present several different molecular subtypes concurrently (18). The study by Cho et al. highlights several differences between C-GBMs and S-GBMs and importantly has identified differential response to drug screening in vitro and a potential for targeting of nuclear receptors; representing a step forward in the field of understanding this aggressive and currently poorly treated disease process. It is our hope that future studies will begin multiinstitutional efforts to delineate the molecular and genetic profiles of C-GBM with greater numbers of patients to discern reliable and generalizable findings to support preclinical therapeutic development.

\section{Acknowledgments}

Funding: None.

\section{Footnote}

Provenance and Peer Review: This article is commissioned and reviewed by the Section Editor Xian-Xin Qiu [MD, $\mathrm{PhD}$ candidate, Shanghai Proton and Heavy Ion Center (SPHIC), a.k.a. the Proton and Heavy Ion Center of Fudan University Shanghai Cancer Center (FUSCC), Shanghai, China].

Conflicts of Interest: Both authors have completed the ICMJE uniform disclosure form (available at http://dx.doi. org/10.21037/tcr.2019.08.24). The authors have no conflicts of interest to declare.

Ethical Statement: The authors are accountable for all aspects of the work in ensuring that questions related to the accuracy or integrity of any part of the work are appropriately investigated and resolved.

Open Access Statement: This is an Open Access article distributed in accordance with the Creative Commons Attribution-NonCommercial-NoDerivs 4.0 International License (CC BY-NC-ND 4.0), which permits the noncommercial replication and distribution of the article with the strict proviso that no changes or edits are made and the original work is properly cited (including links to both the formal publication through the relevant DOI and the license). See: https://creativecommons.org/licenses/by-nc-nd/4.0/.

\section{References}

1. Kuroiwa T, Numaguchi Y, Rothman MI, et al. Posterior fossa glioblastoma multiforme: MR findings. AJNR Am J Neuroradiol 1995;16:583-9. 
2. Stark AM, Nabavi A, Mehdorn HM, et al. Glioblastoma multiforme-report of 267 cases treated at a single institution. Surg Neurol 2005;63:162-9; discussion 169.

3. Adams H, Chaichana KL, Avendano J, et al. Adult cerebellar glioblastoma: understanding survival and prognostic factors using a population-based database from 1973 to 2009. World Neurosurg 2013;80:e237-43.

4. Tauziède-Espariat A, Saffroy R, Pagès M, et al. Cerebellar high-grade gliomas do not present the same molecular alterations as supratentorial high-grade gliomas and may show histone $\mathrm{H} 3$ gene mutations. Clin Neuropathol 2018;37:209-16.

5. Babu R, Sharma R, Karikari IO, et al. Outcome and prognostic factors in adult cerebellar glioblastoma. J Clin Neurosci 2013;20:1117-21.

6. Takahashi Y, Makino K, Nakamura H, et al. Clinical characteristics and pathogenesis of cerebellar glioblastoma. Mol Med Rep 2014;10:2383-8.

7. Utsuki S, Oka H, Miyajima Y, et al. Adult cerebellar glioblastoma cases have different characteristics from supratentorial glioblastoma. Brain Tumor Pathol 2012;29:87-95.

8. Cho HJ, Zhao J, Jung SW, et al. Distinct genomic profile and specific targeted drug responses in adult cerebellar glioblastoma. Neuro Oncol 2019;21:47-58.

9. Nomura M, Mukasa A, Nagae G, et al. Distinct molecular profile of diffuse cerebellar gliomas. Acta Neuropathol 2017;134:941-56.

10. Tsung AJ, Prabhu SS, Lei X, et al. Cerebellar glioblastoma: a retrospective review of 21 patients at a single institution. J Neurooncol 2011;105:555-62.

11. Stark AM, Maslehaty H, Hugo HH, et al. Glioblastoma of the cerebellum and brainstem. J Clin Neurosci 2010;17:1248-51.

12. Weber DC, Miller RC, Villa S, et al. Outcome and prognostic factors in cerebellar glioblastoma multiforme in adults: a retrospective study from the Rare Cancer Network. Int J Radiat Oncol Biol Phys 2006;66:179-86.

13. Djalilian HR, Hall WA. Malignant gliomas of the cerebellum: an analytic review. J Neurooncol 1998;36:247-57.

14. Tenga A, Beard JA, Takwi A, et al. Regulation of Nuclear Receptor Nur77 by miR-124. PLoS One 2016;11:e0148433.

15. Chang LF, Lin PC, Ho LI, et al. Overexpression of the orphan receptor Nur77 and its translocation induced by PCH4 may inhibit malignant glioma cell growth and induce cell apoptosis. J Surg Oncol 2011;103:442-50.

16. Bakas S, Akbari H, Sotiras A, et al. Advancing The Cancer Genome Atlas glioma MRI collections with expert segmentation labels and radiomic features. Sci Data 2017;4:170117.

17. Hu LS, Ning S, Eschbacher JM, et al. Radiogenomics to characterize regional genetic heterogeneity in glioblastoma. Neuro Oncol 2017;19:128-37.

18. Patel AP, Tirosh I, Trombetta JJ, et al. Single-cell RNAseq highlights intratumoral heterogeneity in primary glioblastoma. Science 2014;344:1396-401.
Cite this article as: Tora MS, Adamson DC. Discernable differences in the genetic and molecular profile of cerebellar glioblastoma. Transl Cancer Res 2019;8(Suppl 6):S553-S558. doi: $10.21037 /$ tcr.2019.08.24 